\title{
Detecting Extra-solar Planetary Transits with a Schmidt
}

\author{
Ian R. Parry
}

\author{
AAO, PO Box 296, Epping, NSW 2121, Australia
}

Fred G. Watson

RGO, Madingley Road, Cambridge, CB3 OEZ, UK

B. Esperanza Carrasco

INAOE, Apartado Postal 51, 72000 Pue, Puebla, Mexico

\begin{abstract}
We describe an instrumental configuration for detecting large gas-giant planets orbiting main sequence stars via the small drop in stellar brightness that occurs when the planet transits the stellar disk. Our proposed scheme involves the long-term monitoring of the light-curves of tens of thousands of stars using a Schmidt telescope coupled to a CCD via a bundle of 10,000 optical fibres. Using an existing theoretical model of planetary system formation we calculate a detection rate of 14 transits per year for our proposed system.
\end{abstract}

\section{Introduction}

The idea of detecting an extra-solar planet by observing the small drop in light intensity when the planet transits its parent star is not a new one and it has long been recognised that to detect such an event requires an extremely intensive observing program. Borucki and Summers (1984) estimated the transit rate for Jupiter-like planets to be $2 \times 10^{-7}$ transits per star per night assuming that all stars have a Jupiter-sized planet in a Jupiter-sized orbit. They concluded that $\sim 10,000$ stars would have to be monitored continuously by a $1 \mathrm{~m}$ class telescope and they suggested that the wide field of view of the telescope would have to be coupled to the detector by optical fibres. With such a set up they estimated they would detect $\sim 1$ planetary transit per year. In this paper we present our own, more detailed transit rate predictions and consider how these can be tested observationally using multi-fibre photometry on a Schmidt.

\section{Transit Rate Predictions}

Our predictions are based on the theory of planetary system formation due to Hughes (1992) which gives the size of the orbit of the nearest gas-giant as a function of the mass of the parent star: 


$$
\log (r)=1.64 \log \left(M_{*}\right)+0.69
$$

where $r$ is the orbital radius in $\mathrm{AU}$ and $M_{*}$ is the mass of the star in solar units.

We only consider the nearest gas-giant in each system because transits by terrestrial-sized planets are undetectable and including gas-giant planets in larger orbits in the calculations does not significantly increase the predicted transit rate. The predictions are also limited to main sequence stars with $6<M_{V}<$ 12 because their smaller planetary orbits and deeper transit light curves make observable transits more likely. The calculations assume Jupiter-sized planets and orbits randomly oriented to the line of sight.

Table 1 shows the predictions based on the model as a function of $M_{V}$ for $M_{V} \pm 0.5$ magnitude bins. [ $\mathrm{s} / \mathrm{n} \mathrm{rat}$ ] is the signal-to-noise ratio required to make a $3 \sigma$ detection of the transit and [Tr depth] is the fractional depth of the observed transit. [ $\operatorname{Tr}$ len] is the length of the transit in hours. [Orb per] is the orbital period of the planet in years. [ $\mathrm{Tr}$ rate] is the number of transits expected per star per night.

\section{Photometry with Fibres}

Borucki et al. (1988) presented evidence that the throughput of a fibre depends on where on the input face the light hits (see figure 6 of their paper) and therefore photometry of sufficient accuracy would not be possible. However, we have performed similar laboratory experiments and we find that for most of the central area of a fibre's input face there is no positional dependance on throughput (Parry et al. 1994).

One of us (FGW), in collaboration with Richard Robinson, has also performed some fibre photometry experiments on the UKST which were primarily

Table 1 - Predictiong for the UKST

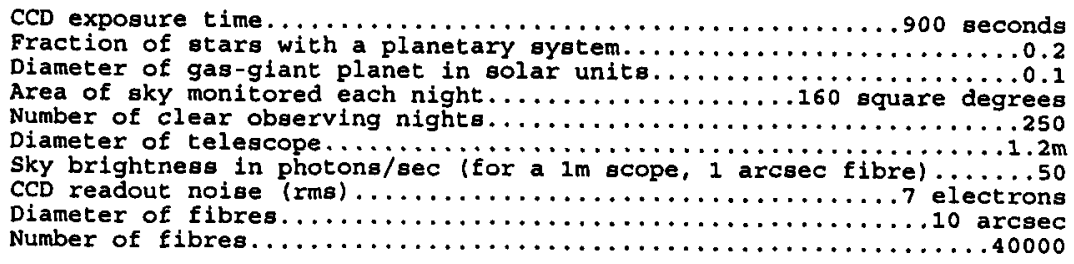

$\begin{array}{lccccccc}\text { Mv } & 6.00 & 7.00 & 8.00 & 9.00 & 10.00 & 11.00 & 12.00 \\ \text { s/n rat } & 227.6 & 178.7 & 140.3 & 110.2 & 80.7 & 52.1 & 32.9 \\ \text { Tr depth } & 0.013 & 0.017 & 0.021 & 0.027 & 0.037 & 0.058 & 0.091 \\ \text { Tr len } & 23.646 & 19.900 & 16.748 & 14.095 & 11.292 & 8.429 & 6.220 \\ \text { Orb per } & 7.6 & 5.5 & 4.0 & 2.9 & 2.0 & 1.2 & 0.8 \\ \text { Tr rate } & 0.909 \mathrm{E}-07 & 0.146 \mathrm{E}-06 & 0.232 \mathrm{E}-06 & 0.331 \mathrm{E}-06 & 0.531 \mathrm{E}-06 & 0.857 \mathrm{E}-06 & 0.140 \mathrm{E}-05 \\ & & & & & & & \\ \text { counts } & 0.692 \mathrm{E}+07 & 0.680 \mathrm{E}+07 & 0.670 \mathrm{E}+07 & 0.664 \mathrm{E}+07 & 0.659 \mathrm{E}+07 & 0.654 \mathrm{E}+07 & 0.652 \mathrm{E}+07 \\ \text { lim mag } & 19.17 & 19.54 & 19.91 & 20.28 & 20.72 & 21.30 & 21.90 \\ \text { n stars } & 0.463 \mathrm{E}+07 & 0.165 \mathrm{E}+07 & 0.907 \mathrm{E}+06 & 0.445 \mathrm{E}+06 & 0.326 \mathrm{E}+06 & 0.246 \mathrm{E}+06 & 0.215 \mathrm{E}+06 \\ \text { transitg } & 0.91 & 1.46 & 2.32 & 3.31 & 5.31 & 8.57 & 14.03 \\ \text { Probab } & 0.597 \mathrm{E}+00 & 0.767 \mathrm{E}+00 & 0.901 \mathrm{E}+00 & 0.964 \mathrm{E}+00 & 0.995 \mathrm{E}+00 & 0.100 \mathrm{E}+01 & 0.100 \mathrm{E}+01\end{array}$


to test the feasibility of using multi-fibre photometry to record activity in known flare stars. 10 fibres with $200 \mu \mathrm{m}$ (13 arcsec) core diameters were used to couple the focal plane to a CCD. A typical light curve from a fibre placed on a flare star when no flaring was occurring (i.e. the light from the star was constant) shows no systematic variations due to the instrumentation and the signal-to-noise ratio obtained is entirely due to shot noise (Parry et al. 1994). For each time epoch the photometered value was normalized using all the measurements made at that time (ensemble photometry).

\section{Proposed System}

Clearly, our predicted transit rates show that an extremely intensive observing program has to be carried out to have a good chance of detecting a planet. This requires a Schmidt with an aperture of at least $60 \mathrm{~cm}$ dedicated entirely to this project and $\sim 10,000$ stellar light curves have to be obtained each night for about a year. Given the practical constraints of using very large numbers of fibres and the large numbers of light-curves demanded by the transit-rate predictions we propose the following observational system.

- A dedicated Schmidt telescope.

- 4-8 fibre modules that can be quickly loaded in to the focal plane of the telescope (for all-year operation).

- 40,000 fibres of 10 arcsec diameter permanently mounted in each module.

- Each module is split into 4 bundles of 10,000 fibres.

- Each bundle of 10,000 is used for a different field so the telescope continuously cycles through the 4 fields (this effectively increases the field coverage by $\times 4)$.

- Each of the 4 bundle outputs is arranged as a $100 \times 100$ array and is reimaged on to a $1024 \times 1024$ thinned Tektronics CCD. (Only one subbundle of 10,000 fibres is active at any one time.)

- The CCD has a readout noise of $7 \mathrm{e}^{-} \mathrm{rms}$, a gain of $5.5 \mathrm{e}^{-/ A D U}$ and a full well capacity of $370 \mathrm{ke}^{-}$.

- Coherent fibre bundles are used for auto-guiding.

Table 1 shows the results assuming the transit-rate predictions and an instrumental configuration like the one described here, on the UKST. The starting assumptions are shown at the top. We have assumed that 250 clear nights corresponds to 1 year of elapsed time. [counts] is the total number of photons detected for a star at the limiting magnitude including the contribution from the sky (which is $6.48 \times 10^{6}$ photons). [lim mag] is the limiting magnitude to obtain the required signal-to-noise ratio in the $\mathrm{V}$ band. The stars are of course much brighter in the $R$ and $I$ bands. [n stars] is the number of stars in the monitored area of sky that are brighter than the limiting magnitude. [transits] 
is the number of transits expected during the survey based on either the number of stars in the monitored area or the number of fibres (whichever is the smaller). Finally, [probab] is the probability that at least one transit is seen in the survey.

\section{Conclusions}

A dedicated Schmidt telescope coupled to a CCD with tens of thousands of optical fibres can place strong constrain ts on the number of extra-solar planetary systems and theories of planetary system formation-even if no transits are observed. The biggest two problems to overcome are getting the observing time on a large Schmidt and selection of the fields. The predictions clearly show that the best stars to observe are dwarfs with masses less than that of the Sun. The problem is how do we select such a sample of dwarfs? Selection on the basis of colour will allow a sample of cool stars to be selected easily but this could still be significantly contaminated by cool giants. The contamination by giants can possibly be reduced by choosing fields at the galactic poles or in the direction of optically thick dark clouds so that only foreground objects can be seen. There are no major technical or instrumental problems to solve in the sense that fibres and CCDs are now fairly well understood.

If an existing Schmidt telescope is used the total cost of the project including the CCD and the operational costs is likely to be less than US $\$ 1 \mathrm{M}$. Before such a large project is undertaken some pilot studies should be carried out. These should include a run on a Schmidt with $\sim 1000$ fibres and some laboratory tests to evaluate different fibre types including the cheaper PCS and HCS types.

Finally, apart from the main objective of detecting gas-giant planets, our proposed survey would generate a great deal of other interesting data on: dwarf binary systems including widely separated systems; possible brown dwarf detections; variability at the faint end of the main sequence; stellar spots; flare stars; and possible short duration MACHO events. The technique could also be used to carry out a search for supernovae in faint galaxies.

\section{Acknowledgments}

We would like to thank Richard Robinson for the major part he played in the UKST fibre photometry run. We also wish to thank the Physics Department of Durham University, UK where two of us (IRP and BEC) were working during the earlier part of this project.

\section{References}

Borucki W. J. \& Summers A. L., 1984, Icarus, 58, 121

Borucki W. J., Torbet E. B. \& Pham P. C., 1988, in Fiber Optics in Astronomy, ASP Conf. Ser. 3, S. C. Barden, ed., p.247

Hughes D. W.,1992, New Scientist, Dec 12, p.29

Parry I. R., Carrasco B. E. \& Watson F. G., 1994, Proc of SPIE, Vol. 2198, in press 


\section{Discussion}

Florentin-Nielsen: Is it at all possible to utilize Fabry lenses in front of the fibres to improve on the poor photometric properties of some fibres?

Parry: Yes, this has been tried in some spectroscopic systems, more to change the f/ratio than to improve photometric properties.

Steel: Has the effect of varying intensity from the system (star plus planets), as the planets orbit the star, been factored in? For example, a single planet with albedo $\sim 0.5$ will contribute an intensity varying from $\sim 50 \%$ to $0 \%$ of the intensity drop in transit as it moves from phase $180^{\circ}$ to $0^{\circ}$.

Parry: No, the model is simple and treats the planet as being perfectly opaque. 